\title{
CT Diagnosis of Scleral Rupture
}

\author{
LARRY BENJAMIN $†$ and RICHARD WORMALD* \\ London
}

\begin{abstract}
Summary
The diagnosis of scleral rupture by computerised tomography (CT) in two cases is reported. In both cases we were able to confirm the CT findings at surgery. The CT appearances of scleral rupture are illustrated.
\end{abstract}

The diagnosis of posterior scleral rupture can be difficult. ${ }^{1}$ It may be made on clinical grounds, with a history of severe blunt trauma and the findings of a soft eye with reduced visual acuity. Eye movements are often painful and restricted, and this, combined with the site of maximal chemosis may indicate the quadrants of the globe involved in the rupture.

Haemorrhage in the anterior and posterior segments may be extensive and often prevents visualization of the rupture site.

We present two cases in which CT was used to demonstrate scleral rupture and confirm the clinical suspicion after ultrasound had failed to reveal a scleral defect.

\section{Case Reports}

Case 1

A 39-year-old man was referred to Moorfields Eye Hospital with a history of having been hit in the left eye by a squash ball. He could barely perceive light with the affected eye and projection was inaccurate. The lids were oedematous and bruised, the conjunctiva grossly chemosed, the cornea decompensated and the anterior chamber full of blood. There was an afferent pupillary defect (diagnosed consensually) and the intraocular pressure was $8 \mathrm{mmHg}$, half that of the fellow eye. Downgaze was limited and painful.

Ultrasonography showed a choroidal detachment and intragel haemorrhage but scleral rupture could not be demonstrated. However, CT
(EMI 5005 and GE 9800) showed a scleral discontinuity in the upper temporal quadrant and also demonstrated that the lens was dislocated (Figs. 1, 2 and 3 ).

After primary repair the retina detached, but following a vitreolensectomy and several gas-fluid exchanges, the eye was normotensive, the retina flat and the visual acuity improved to $6 / 12$ with a contact lens.

\section{Case 2}

A 49-year-old man presented to Moorfields Eye Hospital after being punched in his right eye. He had previously suffered from a sclerosing pseudotumour involving the right orbit, which had failed to respond to treatment, causing progressive visual loss and proptosis. The eye was blind to light with a total afferent pupillary defect and a complete tarsorrhaphy had been performed to protect his cornea. Since the injury, the patient complained of severe pain on moving the eye. The globe was soft and painful on palpation through the closed lids. Clinically it was suspected that the globe might be ruptured but ultrasound showed only a suprachoroidal opacity and intragel haemorrhage. CT scan (5005) was therefore performed and although the interpretation was made more difficult by the established changes of the pseudotumour, it appeared to show discontinuity of the anterior nasal portion of the globe in coronal sections (Figs. 4 and 5). The soft tissue opacity around the globe showed the extent of the pre-existing pseudotumour.

Berause the eye was blind and because of the risk of sympathetic involvement of the othereye, it was

Correspondence to: R. Wormald, Moorfields Eye Hospital, City Road, London EC1.

$\dagger$ Registrar, Moorfields Eye Hospital. * ${ }^{*}$ Senior Registrar, Moorfields Eye Hospital. 


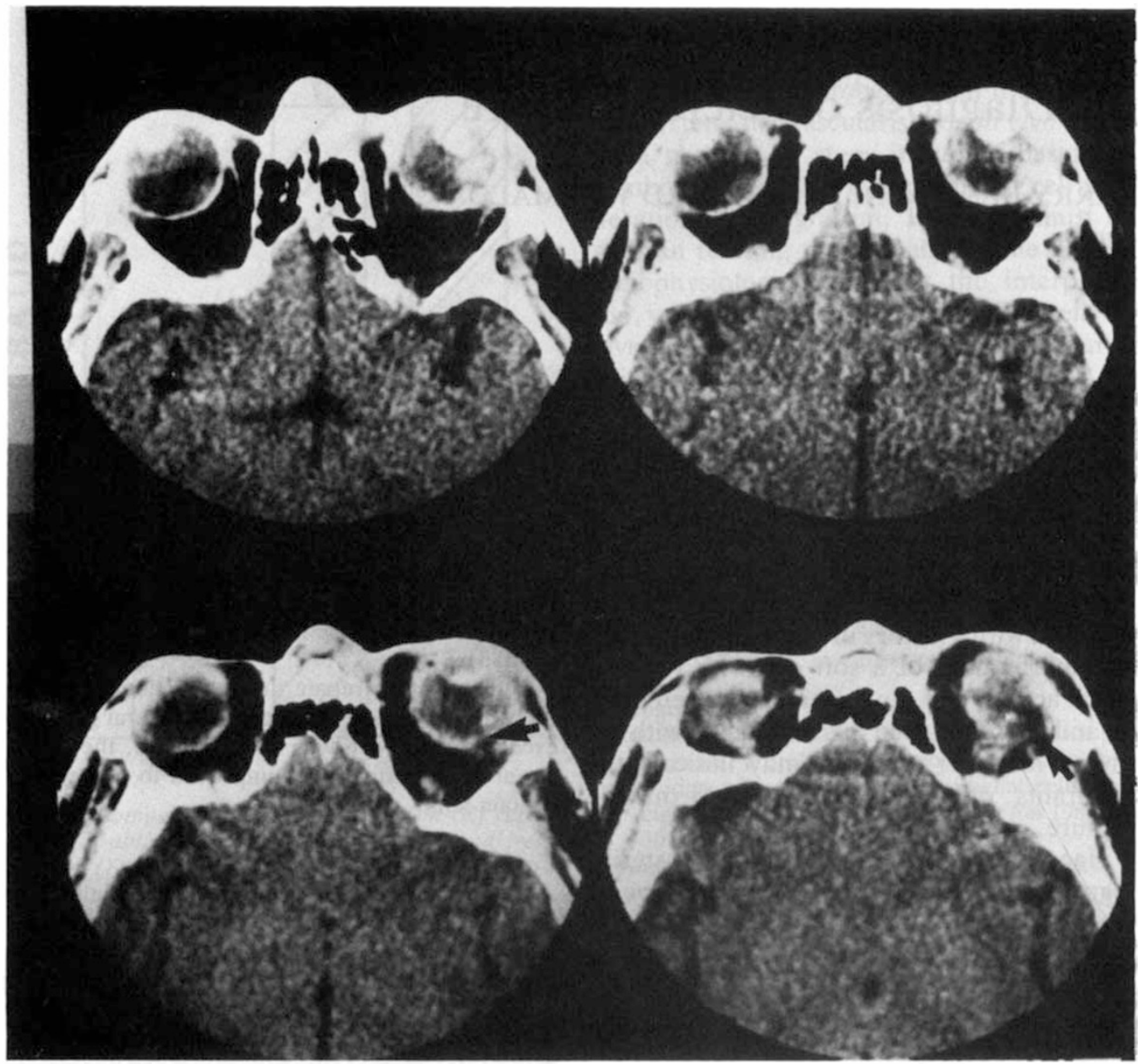

Fig. 1. Axial CT showing extensive intraocular haemorrhage and site of superotemporal scleral rupture (arrows).

possible to advise removal of the eye and obtain informed consent. When the tarsorrhaphy was opened at surgery, a rupture of the globe extending across the cornea to the nasal equator was confirmed; the eye was enucleated.

\section{Discussion}

These two cases underline the value of the relatively accurate anatomical assessment of the orbital and ocular structures afforded by $\mathrm{CT}$. In the first, it was possible to plan primary repair of the globe with an idea of the localisation and extent of the scleral rupture. It was also useful to have forewarning of the upward dislocation of the lens when planning the subsequent vitrectomy. Lensectomy was obviously necessary and appropriate informed consent was obtained before surgery. Similar advantages are illustrated by the second case.

Groves $^{2}$ has reported the value of CT in assessment of severe ocular trauma. Although his cases illustrate mainly penetrating trauma, he did show that CT can define scleral discontinuity and gave one example of scleral rupture following blunt trauma.

A useful synopsis of changes found on CT following trauma was given by Cobb et al. ${ }^{3}$ 


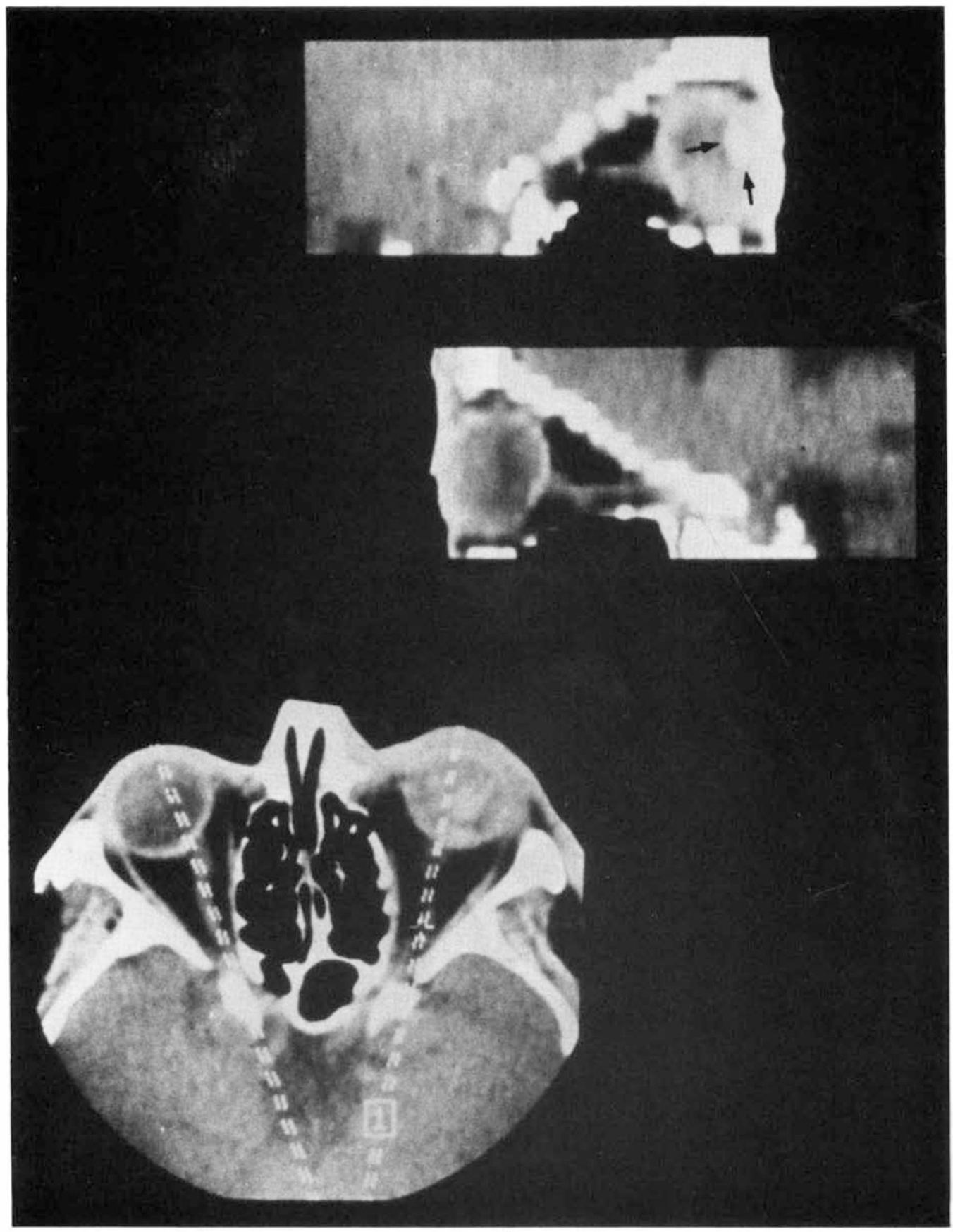

Fig. 2. Paraxial reformat in plane of optic nerve shows upward dislocation of the crystalline lens (arrows). 


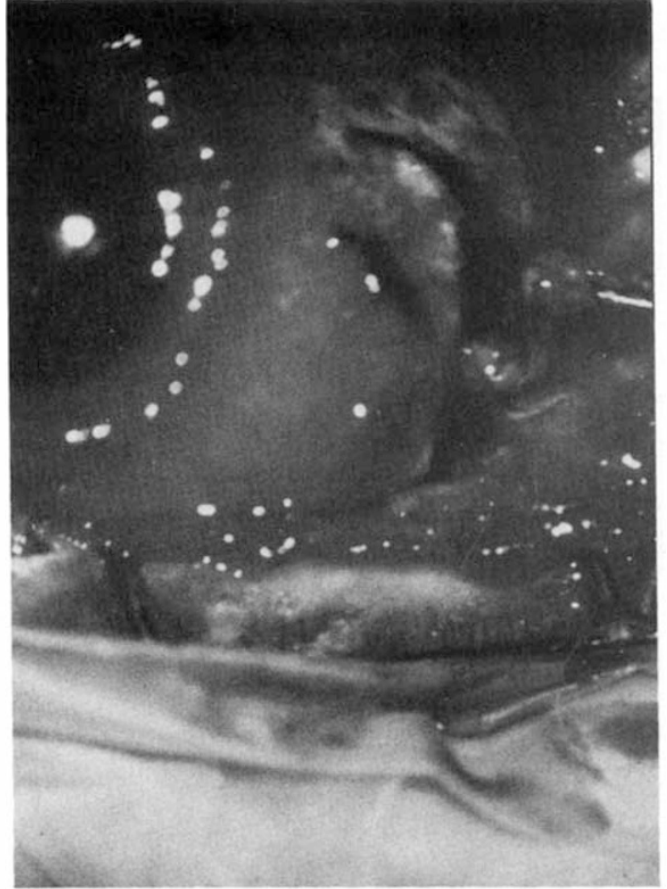

Fig. 3. Per-operative photograph showing rupture site.
Among the changes they described were those of scleral rupture: they suggested that air in the vitreous cavity is a pathognomonic sign, but this will be absent in many cases, since it depends on anterior disruption of the globe, or on air within the orbit, as may occur with a penetrating injury or a concurrent orbital blow-out fracture. The most specific sign must be scleral discontinuity if this can be positively identified (Fig. 1).

Cherry ${ }^{4}$ described the common sites of scleral rupture following blunt ocular trauma and noted that the only hope of restoring useful vision was by attempting reparative surgery. Modern vitrectomy instrumentation has facilitated secondary repair. Although others ${ }^{5}$ have suggested that ultrasound and electrodiagnosis are useful in preoperative assessment of these patients, we found ultrasound unhelplful and electrodiagnosis irrelevant in our cases. Clearly it is important to define posterior scleral rupture accurately and to assess its extent as an aid to reparative surgery; CT is currently the best available method.

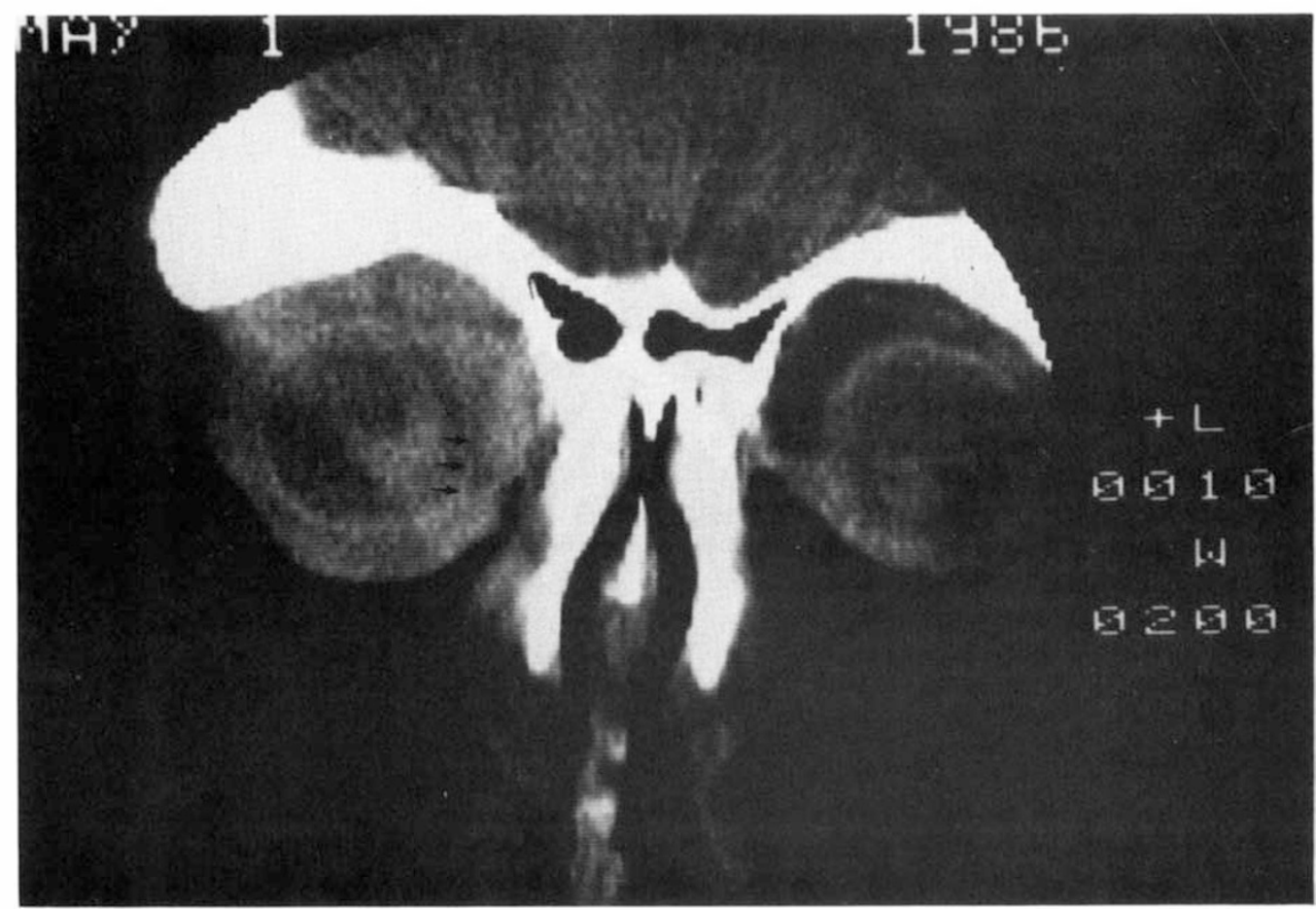

Fig. 4. Coronal CT (5005) showing intraocular haemorrhage and probable site of nasal rupture (arrows). 


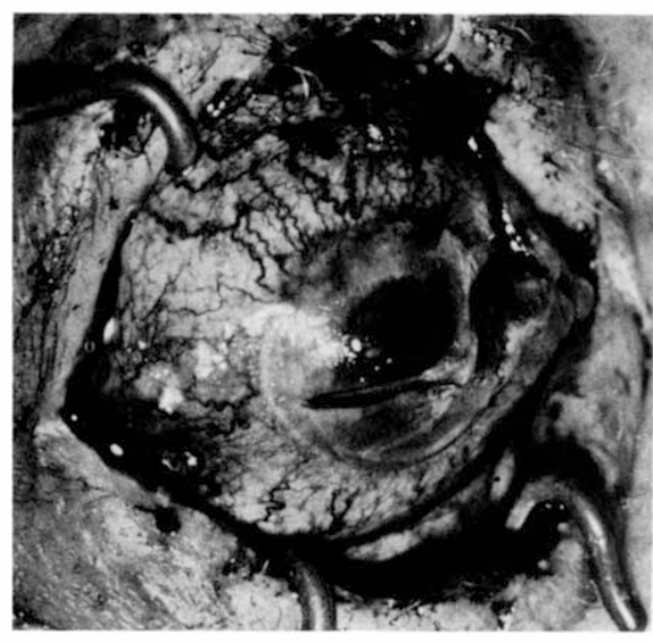

Fig. 5. Per-operative photograph after opening the tarsorrhaphy showing the corneo-scleral rupture.

In this paper we wish to stress the value of the CT scan in the assessment of patients with suspected scleral rupture posteriorly or, as in the second case, when examination of the integrity of the globe is precluded.

We would like to thank Mr John Wright FRCS and Mr Zdenek Gregor FRCS for allowing us to report on their patients and also to express appreciation to $\mathrm{Mr}$ Gregor and Dr Ivan Mosely FRCR for advice and encouragement.

\section{References}

1 Paton D and Goldberg MS: In Management of ocular injuries. Printed by W. B. Saunders, Philadelphia, 1976.

${ }^{2}$ Groves AS: Orbital trauma and computed tomography. Ophthalmology 1980, 87: 403-11.

${ }^{3}$ Cobb SR, et al.: Computed tomographic evaluation of ocular trauma. Computerized Radiol 1985, 9: 1-10.

${ }^{4}$ Cherry PMH: Indirect traumatic rupture of the globe. Arch Ophthalmol 1978, 96: 252-6.

${ }^{5}$ Crews SJ, Hillman JS and Thompson CRS: Electrodiagnosis and Ultrasonography in the assessment of recent major trauma. Trans Ophthalmol Soc UK 1975, 95: 315-21. 